\title{
Truncated Weibull power Lomax distribution: statistical properties and applications
}

\section{Sanaa Al-Marzouki}

Statistics Department, Faculty of Science, King AbdulAziz University, Jeddah, Kingdom of Saudi Arabia.

\begin{abstract}
A new four-parameter distribution, called the truncated Weibull power Lomax (TWPL) distribution is introduced. We calculate the density (pdf), distribution function (cdf), quantile function, $\mathrm{r}^{\text {th }}$ moment, inequality measures, and order statistics. Maximum Likelihood methods to estimate the TWPL distribution parameters are proposed. Two real data sets are applied to illustrate the flexibility of the TWPL model compared with some Known distributions.
\end{abstract}

Keywords: Power Lomax distribution, truncated Weibull-G family, moments, order statistics.

2010 MSC: 60E05, 62E10, 62N05.

(C)2019 All rights reserved.

\section{Introduction}

The Power Lomax (PL) distribution is studied by Rady et al. [12]. The PL distribution is a generalization of Lomax (L) distribution. The cdf and pdf of the PL distribution are given, respectively, by

$$
G(x)=1-\left(1+\frac{x^{\beta}}{\gamma}\right)^{-\alpha}, \quad x>0, \alpha, \beta, \gamma>0,
$$

and

$$
g(x)=\frac{\alpha \beta}{\gamma} x^{\beta-1}\left(1+\frac{x^{\beta}}{\gamma}\right)^{-\alpha-1}, \quad x>0, \alpha, \beta, \gamma>0,
$$

where, $\gamma$ is a scale parameter and $\alpha$ and $\beta$ are shape and scale parameters, respectively.

In the last years, many statisticians are interested to expand generating family in order to obtain better fit for data analyzing. Some known generating family are: The the beta-G by Eugene et al. [6], Logistic-G introduced by Torabi and Montazeri [14], exponentiated Weibull-G studied by Hassan and Elgarhy [9], Kumaraswamy Weibull-G by Hassan and Elgarhy [8], Garhy-G of Elgarhy et al. [5], Type II half logistic-G by Hassan et al. [10], Najarzadegan et al. [11] proposed a new truncated Weibull-G (TW-G), odd Fréchet-G

Email address: salmarzouki@kau.edu.sa (Sanaa Al-Marzouki)

doi: $10.22436 /$ jnsa.012.08.05 
proposed by Haq and Elgarhy [7], Muth-G studied by Almarashi and Elgarhy [2], and Elbatal et al. [4] proposed a new alpha power transformation family of distributions among others.

The cdf of TW-G family is given by

$$
F(x ; \theta, \lambda)=\left(1-e^{-\theta}\right)^{-1}\left(1-e^{-\theta(G(x))^{\lambda}}\right), x \in R, \quad \theta, \lambda>0 .
$$

We are interested to make $\theta=1$, then the equation (1.3) becomes

$$
F(x ; \lambda)=A\left(1-e^{-(G(x))^{\lambda}}\right), x \in R, \lambda>0 .
$$

The corresponding pdf to (1.4) is

$$
f(x ; \lambda)=A \lambda g(x)(G(x))^{\lambda-1} e^{-(G(x))^{\lambda}}, x \in R, \lambda>0,
$$

where $A=\left(1-e^{-1}\right)^{-1}, \lambda$ is the shape parameter, and $G($.$) is cdf of any baseline distribution.$

The aim of this paper is to propose a new four parameter distribution based on the TW-G family. The TWPL model provides more flexible model. We wish that the TWPL model will attract wider applications in some areas.

The TWPL model with vector parameters $\varphi$, where $\varphi=(\alpha, \beta, \gamma, \lambda)$, by inserting (1.1) and (1.2) in (1.4) and (1.5) then, the cdf and pdf of TWPL are

$$
F(x ; \varphi)=A\left(1-e^{-\left(1-\left(1+\frac{x^{\beta}}{\gamma}\right)^{-\alpha}\right)^{\lambda}}\right), \alpha, \beta, \gamma, \lambda, x>0
$$

and

$$
f(x ; \varphi)=\frac{A \alpha \lambda \beta}{\gamma} x^{\beta-1}\left(1+\frac{x^{\beta}}{\gamma}\right)^{-\alpha-1}\left(1-\left(1+\frac{x^{\beta}}{\gamma}\right)^{-\alpha}\right)^{\lambda-1} e^{-\left(1-\left(1+\frac{x^{\beta}}{\gamma}\right)^{-\alpha}\right)^{\lambda}} .
$$

The sf and the hrf are, respectively, given by

$$
\mathrm{R}(x ; \varphi)=1-\mathrm{A}\left(1-\mathrm{e}^{-\left(1-\left(1+\frac{\mathrm{x}^{\beta}}{\gamma}\right)^{-\alpha}\right)^{\lambda}}\right)
$$

and

$$
h(x ; \varphi)=\frac{\frac{A \alpha \lambda \beta}{\gamma} x^{\beta-1}\left(1+\frac{x^{\beta}}{\gamma}\right)^{-\alpha-1}\left(1-\left(1+\frac{\chi^{\beta}}{\gamma}\right)^{-\alpha}\right)^{\lambda-1} e^{-\left(1-\left(1+\frac{x^{\beta}}{\gamma}\right)^{-\alpha}\right)^{\lambda}}}{1-A\left(1-e^{-\left(1-\left(1+\frac{\chi^{\beta}}{\gamma}\right)^{-\alpha}\right)^{\lambda}}\right)} .
$$

Figure 1 displays some plots of the pdf and hrf for the TWPL pdf for some different values of parameters.

As seen from Figure 1, TWPL distribution can be uni-model, decreasing, and right skewed. In addition, the hrf of TWPL distribution can be J-shaped, decreasing, and increasing.

The rest of the paper can be organized as follows. In the next section, some mathematical properties of the TWPL distribution is studied. The maximum likelihood method is calculated to obtain the estimators of the parameters in Section 3. Application to a real data illustrating the performance of the new model is given in Section 4. Finally, Conclusions appear in the Section 5. 

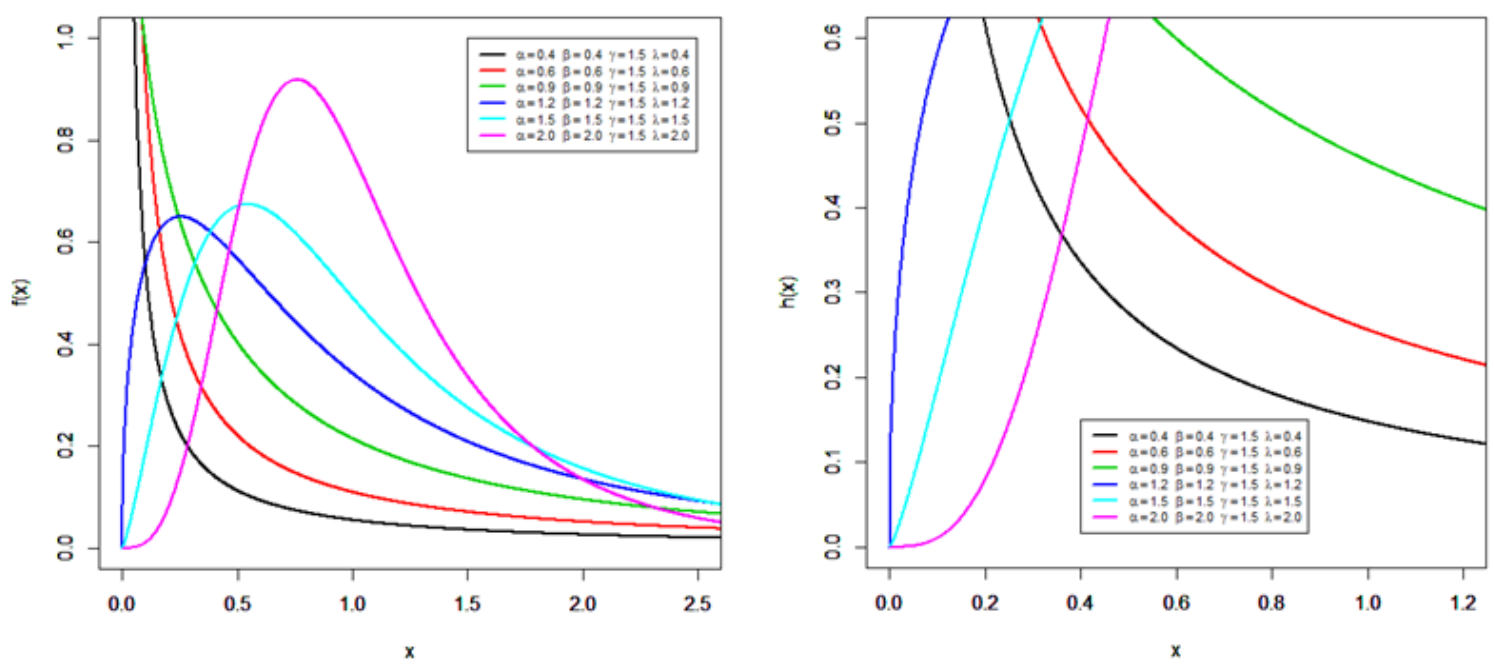

Figure 1: Plots of the pdf and hrf of the TWPL distribution for some different values of parameter.

\section{Some statistical properties}

This section calculates some statistical properties of TWPL model.

\subsection{Quantile function}

The quantile function of the TWPL can be generated by inverting cdf (1.6) as follows

$$
\mathrm{Q}(\mathrm{u})=\sqrt[\beta]{\gamma\left[1-\left(\ln \left(\frac{A}{A-u}\right)\right)^{\frac{1}{\lambda}}\right]^{\frac{-1}{\alpha}}-\gamma}
$$

Simulating the TWL random variable is straightforward. If $\mathrm{U}$ is a uniform variate in the unit interval $(0,1)$, then the random variable $X=Q(u)$ follows $(2.1)$.

\subsection{Useful expansion}

In this subsection expansions of the pdf for TWPL distribution are investigated.

Using the power series for the exponential function, we obtain

$$
e^{-\left(1-\left(1+\frac{x^{\beta}}{\gamma}\right)^{-\alpha}\right)^{\lambda}}=\sum_{i=0}^{\infty} \frac{(-1)^{i}}{i !}\left(1-\left(1+\frac{x^{\beta}}{\gamma}\right)^{-\alpha}\right)^{\lambda i}
$$

By applying (2.2) in (1.7), then,

$$
f(x ; \varphi)=\frac{A \alpha \beta \lambda}{\gamma} \chi^{\beta-1}\left(1+\frac{\chi^{\beta}}{\gamma}\right)^{-\alpha-1} \sum_{i=0}^{\infty} \frac{(-1)^{i}}{i !}\left(1-\left(1+\frac{\chi^{\beta}}{\gamma}\right)^{-\alpha}\right)^{\lambda(i+1)-1} .
$$

Using the generalized binomial theorem, for $\beta>0$ and $|z|<1$,

$$
(1-z)^{\beta-1}=\sum_{i=0}^{\infty}(-1)^{i}\left(\begin{array}{c}
\beta-1 \\
i
\end{array}\right) z^{i}
$$


Then, by applying the binomial expansion (2.4) in (2.3), the pdf of TWPL distribution becomes

$$
f(x ; \varphi)=\frac{\alpha \beta}{\gamma} x^{\beta-1} \sum_{j=0}^{\infty} \eta_{j}\left(1+\frac{x^{\beta}}{\gamma}\right)^{-\alpha(j+1)-1},
$$

where $\eta_{j}=A \lambda \sum_{i=0}^{\infty} \frac{(-1)^{i+j}}{i !}\left(\begin{array}{c}\lambda(i+1)-1 \\ j\end{array}\right)$.

\subsection{Moments}

In this subsection, we intend to derive the moments and the moment generating function of the TWPL model.

By definition, $\mathrm{r}^{\text {th }}$ moment of r.v $\mathrm{X}$ can be achieved from

$$
\mu_{r}^{\prime}=\int_{0}^{\infty} x^{r} f(x ; \varphi) d x
$$

Inserting (2.5) in (2.6), then:

$$
\mu_{r}^{\prime}=\frac{\alpha \beta}{\gamma} \sum_{j=0}^{\infty} \eta_{j} \int_{0}^{\infty} x^{r+\beta-1}\left(1+\frac{x^{\beta}}{\gamma}\right)^{-\alpha(j+1)-1} d x
$$

Setting $y=\frac{x^{\beta}}{\gamma}$, then

$$
\mu_{r}^{\prime}=\alpha \gamma^{r / \beta} \sum_{j=0}^{\infty} \eta_{j} \int_{0}^{\infty} y^{r / \beta}(1+y)^{-\alpha(j+1)-1} d y .
$$

Again make the following transformation $y=\frac{w}{1-w}$, then

$$
\mu_{r}^{\prime}=\alpha \gamma^{r / \beta} \sum_{j=0}^{\infty} \eta_{j} \int_{0}^{1} w^{r / \beta}(1-w)^{\alpha(j+1)-{ }^{r} / \beta^{-1}} d w,
$$

then the $\mathrm{r}^{\text {th }}$ of TWPL becomes

$$
\mu_{r}^{\prime}=\alpha \gamma^{r} / \beta \sum_{j=0}^{\infty} \eta_{j} B(r / \beta+1, \alpha(j+1)-r / \beta) .
$$

The moment generating function (mgf) of the TWPL distribution is

$$
\begin{aligned}
& M_{x}(t)=E\left(e^{t X}\right)=\int_{0}^{\infty} e^{t x} f(x ; \varphi) d x=\int_{0}^{\infty} \sum_{r=0}^{\infty} \frac{t^{r}}{r !} x^{r} f(x ; \varphi) d x=\sum_{r=0}^{\infty} \frac{t^{r}}{r !} E\left(X^{r}\right), \\
& M_{x}(t)=\alpha \gamma / \beta \sum_{i, r=0}^{\infty} \frac{\eta_{j} t^{r}}{r !} B(r / \beta+1, \alpha(j+1)-r / \beta) .
\end{aligned}
$$

\subsection{Order statistics}

The density of the $k^{\text {th }}$ order statistic, for $r=1, \ldots, n$ from independent and identically distributed random variables $X_{1}, X_{2}, \ldots, X_{n}$ is given by

$$
f_{k: n}(x)=\frac{1}{B(k, n-k+1)} f(x) F(x)^{k-1}[1-F(x)]^{n-k} .
$$


Inserting (1.6) and (1.7) in (2.7), then

$$
\begin{aligned}
f_{k: n}(x)= & \frac{\alpha \lambda \beta A^{k}}{\gamma B(k, n-k+1)} x^{\beta-1}\left(1+\frac{x^{\beta}}{\gamma}\right)^{-\alpha-1}\left(1-\left(1+\frac{x^{\beta}}{\gamma}\right)^{-\alpha}\right)^{\lambda-1} e^{-\left(1-\left(1+\frac{x^{\beta}}{\gamma}\right)^{-\alpha}\right)^{\lambda}} \\
& \times\left(1-e^{-\left(1-\left(1+\frac{x^{\beta}}{\gamma}\right)^{-\alpha}\right)^{\lambda}}\right)^{k-1}\left[1-A\left(1-e^{-\left(1-\left(1+\frac{x^{\beta}}{\gamma}\right)^{-\alpha}\right)^{\lambda}}\right)\right]^{n-k} .
\end{aligned}
$$

Setting $r=1$ and $r=n$, in (2.8), we obtain the pdf of the first and largest order statistics of the TWPL distribution.

\section{Maximum likelihood estimators}

The ML estimators of the unknown parameters for the TWPL distribution are determined based on complete samples. Let $X_{1}, \ldots, X_{n}$ be observed values from the TWPL model with set of parameters $\varphi=(\alpha, \beta, \lambda, \gamma)^{\mathrm{T}}$. The total log-likelihood function for the vector of parameters $\varphi$ can be expressed as

$$
\begin{aligned}
\ln L(\varphi)= & n \ln A-n \ln \gamma+\ldots n \ln \beta+n \ln \lambda+n \ln \alpha+(\beta-1) \sum_{i=1}^{n} \ln x_{i}-(\alpha+1) \sum_{i=1}^{n} \ln \left(1+\frac{x_{i}{ }^{\beta}}{\gamma}\right) \\
& +(\lambda-1) \sum_{i=1}^{n} \ln \left(1-\left(1+\frac{x_{i}^{\beta}}{\gamma}\right)^{-\alpha}\right)-\sum_{i=1}^{n}\left(1-\left(1+\frac{x_{i}^{\beta}}{\gamma}\right)^{-\alpha}\right)^{\lambda} .
\end{aligned}
$$

The elements of the score function $\mathrm{U}(\varphi)=\left(\mathrm{U}_{\alpha}, \mathrm{U}_{\beta}, \mathrm{U}_{\lambda}, \mathrm{U}_{\gamma}\right)$ are given by

$$
\begin{aligned}
\frac{\partial \ln \ell}{\partial \alpha}= & \frac{n}{\alpha}-\sum_{i=1}^{n} \ln \left(1+\frac{x_{i}{ }^{\beta}}{\gamma}\right)+(\lambda-1) \sum_{i=1}^{n} \frac{\left(1+\frac{x_{i}^{\beta}}{\gamma}\right)^{-\alpha} \ln \left(1+\frac{x_{i}^{\beta}}{\gamma}\right)}{1-\left(1+\frac{x_{i} \beta}{\gamma}\right)^{-\alpha}} \\
& -\lambda \sum_{i=1}^{n}\left(1-\left(1+\frac{x_{i}^{\beta}}{\gamma}\right)^{-\alpha}\right)^{\lambda-1}\left(1+\frac{x_{i}^{\beta}}{\gamma}\right)^{-\alpha} \ln \left(1+\frac{x_{i}^{\beta}}{\gamma}\right), \\
\frac{\partial \ln \ell}{\partial \beta}= & \frac{n}{\beta}+\sum_{i=1}^{n} \ln x_{i}-\frac{(\alpha+1)}{\gamma} \sum_{i=1}^{n} \frac{x_{i}^{\beta} \ln x_{i}}{1+\frac{x_{i}^{\beta}}{\gamma}}-\frac{\alpha(\lambda-1)}{\gamma} \sum_{i=1}^{n} \frac{\left(1+\frac{x_{i}^{\beta}}{\gamma}\right)^{-\alpha-1} x_{i}^{\beta} \ln x_{i}}{1-\left(1+\frac{x_{i} \beta}{\gamma}\right)^{-\alpha}} \\
& -\frac{\alpha \lambda}{\gamma} \sum_{i=1}^{n}\left(1-\left(1+\frac{x_{i}{ }^{\beta}}{\gamma}\right)^{-\alpha}\right)^{\lambda-1}\left(1+\frac{x_{i}{ }^{\beta}}{\gamma}\right)^{-\alpha-1} x_{i}^{\beta} \ln x_{i}, \\
\frac{\partial \ln \ell}{\partial \lambda}= & \frac{n}{\lambda}+\sum_{i=1}^{n} \ln \left(1-\left(1+\frac{x_{i}{ }^{\beta}}{\gamma}\right)^{-\alpha}\right)-\sum_{i=1}^{n}\left(1-\left(1+\frac{x_{i}^{\beta}}{\gamma}\right)^{-\alpha}\right)^{\lambda} \ln \left(1-\left(1+\frac{x_{i} \beta}{\gamma}\right)^{-\alpha}\right),
\end{aligned}
$$

and

$$
\begin{aligned}
\frac{\partial \ln \ell}{\partial \gamma}= & \frac{-n}{\gamma}+\frac{(\alpha+1)}{\gamma^{2}} \sum_{i=1}^{n} \frac{x_{i}^{\beta}}{1+\frac{x_{i}^{\beta}}{\gamma}}-\frac{\alpha(\lambda-1)}{\gamma^{2}} \sum_{i=1}^{n} \frac{\left(1+\frac{x_{i}^{\beta}}{\gamma}\right)^{-\alpha-1} x_{i}^{\beta}}{1-\left(1+\frac{x_{i}^{\beta}}{\gamma}\right)^{-\alpha}} \\
& +\frac{\alpha \lambda}{\gamma^{2}} \sum_{i=1}^{n} x_{i}^{\beta}\left(1+\frac{x_{i}^{\beta}}{\gamma}\right)^{-\alpha-1}\left(1-\left(1+\frac{x_{i}^{\beta}}{\gamma}\right)^{-\alpha}\right)^{\lambda-1} .
\end{aligned}
$$

The ML estimators of the model parameters are determined by solving numerically the non-linear equations $\partial \ln \ell / \partial \alpha=0, \partial \ln \ell / \partial \beta=0, \partial \ln \ell / \partial \lambda=0$, and $\partial \ln \ell / \partial \gamma=0$, simultaneously. 


\section{Applications}

In this section, we use two real data sets to explain the importance and flexibility of the TWPL model. We compare the fits of the TWPL model with some models namely: the gamma L (GL) (Cordeiro et al., [3]), beta L (BL) (Eugene et al., [6]), and exponentiated L (EL) (Abdul-Moniem and Abdel-Hameed, [1]) distributions.

The maximized log-likelihood $(-\ell)$, Akaike information criterion (AIC), the corrected Akaike information criterion (CAIC), Anderson-Darling $\left(A^{*}\right)$, and Cramér-Von Mises $\left(W^{*}\right)$ statistics are used for model selection.

Example 4.1. The first data set is obtained from Tahir et al. [13] represents Failure times of 63 Aircraft Windshield. The data are: $0.046,1.436,2.592,0.140,1.492,2.600,0.150,1.580,2.670,0.248,1.719,2.717$, $0.280,1.794,2.819,0.313,1.915,2.820,0.389,1.920,2.878,0.487,1.963,2.950,0.622,1.978,3.003,0.900,2.053$, $3.102,0.952,2.065,3.304,0.996,2.117,3.483,1.003,2.137,3.500,1.010,2.141,3.622,1.085,2.163,3.665,1.092$, $2.183,3.695,1.152,2.240,4.015,1.183,2.341,4.628,1.244,2.435,4.806,1.249,2.464,4.881,1.262,2.543,5.140$.

For the first data set, Table 1 gives the MLEs of the fitted models and their standard errors (SEs) in parenthesis. The values of goodness-of-fit statistics are listed in Table 3.

It is noted, from Table 2, that the TWPL distribution provides a better fit than other competitive fitted models. It has the smallest values for goodness-of-fit statistics among all fitted models. Plots of the histogram, epdf, ecdf, esf and pp plot are shown in Figure 2. This figure supported the conclusion drawn from the numerical values in Table 2.

Example 4.2. The second data set is obtained from Tahir et al. [13] and represents failure times of 84 Aircraft Windshield. The data are: $0.040,1.866,2.385,3.443,0.301,1.876,2.481,3.467,0.309,1.899,2.610$, $3.478,0.557,1.911,2.625,3.578,0.943,1.912,2.632,3.595,1.070,1.914,2.646,3.699,1.124,1.981,2.661,3.779$, $1.248,2.010,2.688,3.924,1.281,2.038,2.82,3,4.035,1.281,2.085,2.890,4.121,1.303,2.089,2.902,4.167$, $1.432,2.097,2.934,4.240,1.480,2.135,2.962,4.255,1.505,2.154,2.964,4.278,1.506,2.190,3.000,4.305,1.568$, $2.194,3.103,4.376,1.615,2.223,3.114,4.449,1.619,2.224,3.117,4.485,1.652,2.229,3.166,4.570,1.652,2.300$, $3.344,4.602,1.757,2.324,3.376,4.663$.

Table 3 lists the MLEs of the fitted models and their SEs in parenthesis. The values of goodness-of-fit statistics are presented in Table 4.

Table 1: The MLEs and SEs of the model parameters for Example 4.1.

\begin{tabular}{|l|c|c|c|c|}
\hline Model & \multicolumn{4}{|c|}{ Estimates (SEs) } \\
\hline \multirow{2}{*}{$\operatorname{TWPL}(\alpha, \beta, \lambda, \gamma)$} & $\begin{array}{c}2.718 \\
(2.15)\end{array}$ & $\begin{array}{c}2.433 \\
(0.653)\end{array}$ & $\begin{array}{c}0.659 \\
(0.219)\end{array}$ & $\begin{array}{c}38.778 \\
(23.243)\end{array}$ \\
\hline \multirow{2}{*}{$\mathrm{GL}(a, \alpha, \beta)$} & 1.9073 & 35842.4330 & 39197.5715 & \\
& $(0.3213)$ & $(6945.0743)$ & $(151.6530)$ & \\
\hline $\operatorname{BL}(a, b, \alpha, \beta)$ & 1.9218 & 31.2594 & 4.9684 & 169.5719 \\
& $(0.3184)$ & $(316.8413)$ & $(50.5279)$ & $(339.2067)$ \\
\hline $\operatorname{EL}(a, \alpha, \beta)$ & 1.9145 & 22971.1536 & 32881.9966 & \\
& $(0.3482)$ & $(3209.5329)$ & $(162.2299)$ & \\
\hline
\end{tabular}

Table 2: Goodness-of-fit statistics for Example 4.1.

\begin{tabular}{|c|c|c|c|c|c|}
\hline Model & $-\ell$ & AIC & CAIC & $A^{*}$ & $W^{*}$ \\
\hline TWPL & 101.698 & 211.396 & 212.086 & 0.86988 & 0.14474 \\
\hline GL & 102.8332 & 211.6663 & 212.0731 & 1.112 & 0.1836 \\
\hline BL & -102.9611 & 213.9223 & 214.6119 & 1.1336 & 0.1872 \\
\hline EL & 103.5498 & 213.0995 & 213.5063 & 1.2331 & 0.2037 \\
\hline
\end{tabular}



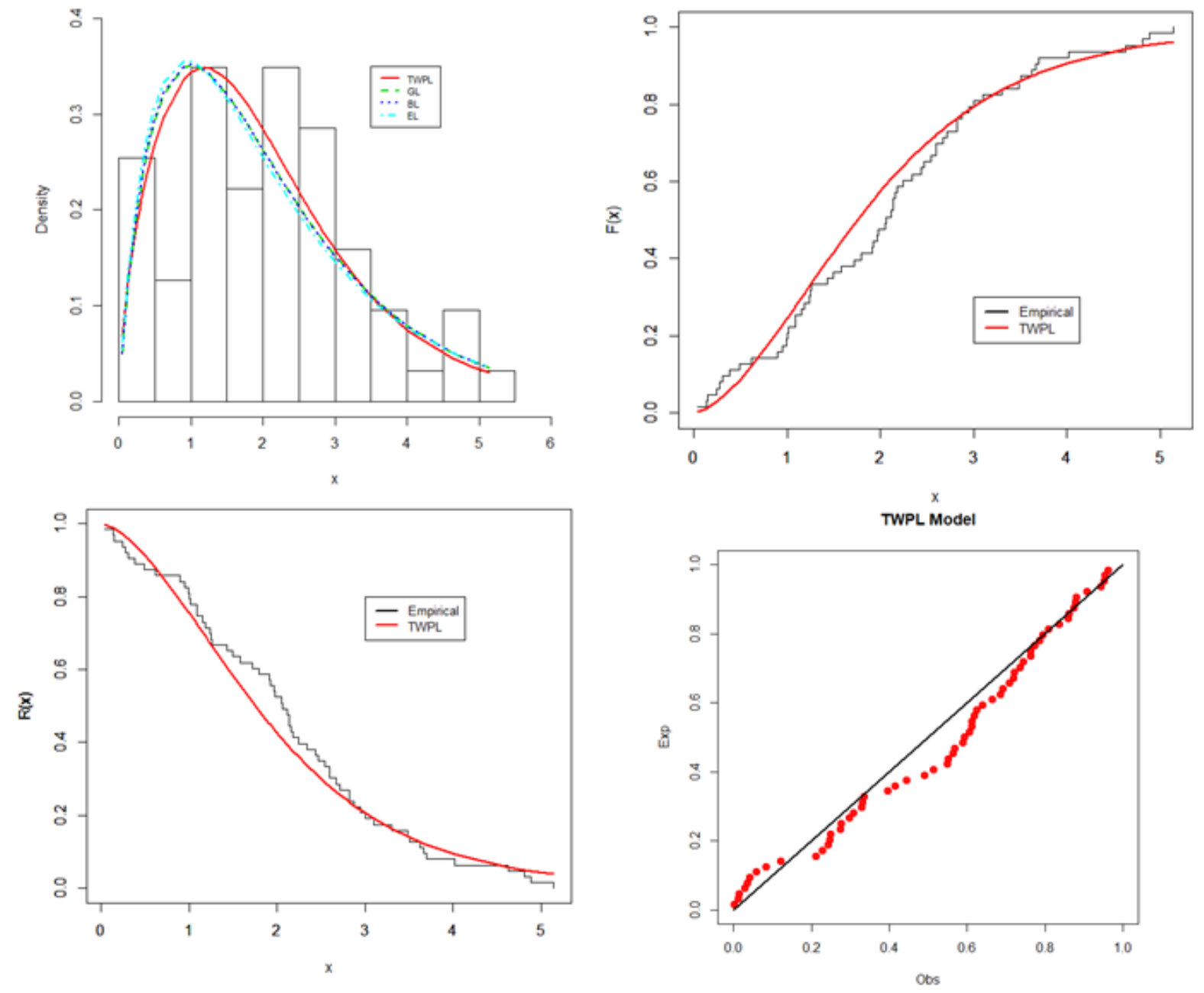

Figure 2: epdf, ecdf, esf, and pp plot of the fitted models for Example 4.1.

Table 3: The MLEs and SEs for Example 4.2.

\begin{tabular}{|l|c|c|c|c|}
\hline Model & \multicolumn{4}{|c|}{ Estimates (SEs) } \\
\hline \multirow{2}{*}{$\operatorname{TWPL}(\alpha, \beta, \lambda, \gamma)$} & 3.916 & 2.723 & 0.936 & 85.847 \\
& $(2.726)$ & $(0.553)$ & $(0.255)$ & $(47.848)$ \\
\hline \multirow{2}{*}{$\operatorname{GL}(a, \alpha, \beta)$} & 3.5876 & 52001.4994 & 37029.6583 & \\
& $(0.5133)$ & $(7955.0003)$ & $(81.1644)$ & \\
\hline $\operatorname{BL}(a, b, \alpha, \beta)$ & 3.6036 & 33.6387 & 4.8307 & 118.8374 \\
& $(0.6187)$ & $(63.7145)$ & $(9.2382)$ & $(428.9269)$ \\
\hline \multirow{2}{*}{$\operatorname{EL}(a, \alpha, \beta)$} & 3.6261 & 20074.5097 & 26257.6808 & \\
& $(0.6236)$ & $(2041.8263)$ & $(99.7417)$ & \\
\hline
\end{tabular}

Table 4: Goodness-of-fit statistics for Example 4.2.

\begin{tabular}{|c|c|c|c|c|c|}
\hline Model & $-\ell$ & AIC & CAIC & $A^{*}$ & $W^{*}$ \\
\hline TWPL & 133.035 & 274.07 & 274.577 & 0.90597 & 0.09641 \\
\hline GL & 138.4042 & 282.8083 & 283.1046 & 1.3666 & 0.1618 \\
\hline BL & 138.7177 & 285.4354 & 285.9354 & 1.4084 & 0.1680 \\
\hline EL & 141.3997 & 288.7994 & 289.0957 & 1.7435 & 0.2194 \\
\hline
\end{tabular}


It is observed, from Table 4, that the TWPL distribution gives a better fit than other fitted models. Plots of the histogram, epdf, ecdf, esf, and pp plot are displayed in Figure 3.


Figure 3: epdf, ecdf, esf, and pp plot of the fitted models for Example 4.2.

\section{Conclusions}

In this paper, we introduce a new four-parameter distribution, called the TWPL distribution. We derive explicit expressions for the quantile, moments, generating functions, and order statistics. We discuss the maximum likelihood estimation of the model parameters. One application illustrate that the TWPL distribution provides consistently better fit than other models.

\section{References}

[1] I. B. Abdul-Moniem, H. F. Abdel-Hameed, On exponentiated Lomax distribution, Int. J. Math. Arch., 3 (2012), 21442150. 4

[2] A. M. Almarashi, M. Elgarhy, A new muth generated family of distributions with applications, J. Nonlinear Sci. Appl., 11 (2018), 1171-1184. 1

[3] G. M. Cordeiro, E. M. M. Ortega, B. V. Popović, The gamma Lomax distribution, J. Stat. Comput. Simul., 85 (2015), 305-319. 4

[4] I. Elbatal, Z. Ahmad, M. Elgarhy, A. M. Almarashi, A New alpha power transformed family of distributions: properties and applications to the Weibull model, J. Nonlinear Sci. Appl., 12 (2019), 1-20. 1 
[5] M. Elgarhy, A. S. Hassan, M. Rashed, Garhy-generated family of distributions with application, Math. Theory Model., 6 (2016), 1-15. 1

[6] N. Eugene, C. Lee, F. Famoye, Beta-normal distribution and its applications, Comm. Statist. Theory Methods, 31 (2002), 497-512. 1, 4

[7] M. Haq, M. Elgarhy, The odd Fréchet-G family of probability distributions, J. Stat. Appl. Prob., 7 (2018), 185-201. 1

[8] A. S. Hassan, M. Elgarhy, A new family of exponentiated Weibull-generated distributions, Int. J. Math. Appl., 4 (2016), 135-148. 1

[9] A. S. Hassan, M. Elgarhy, Kumaraswamy Weibull-generated family of distributions with applications, Adv. Appl. Stat., 48 (2016), 205-239. 1

[10] A. S. Hassan, M. Elgarhy, M. Shakil, Type II half Logistic family of distributions with applications, Pak. J. Stat. Oper. Res., 13 (2017), 245-264. 1

[11] H. Najarzadegan, M. H. Alamatsaz, S. Hayati, Truncated Weibull-G more flexible and more reliable than geta-G distribution, Int. J. Stat. Prob., 6 (2017), 1-17. 1

[12] E. H. A. Rady, W. A. Hassanein, T. A. Elhaddad, The power Lomax distribution with an application to bladder cancer data, SpringerPlus, 5 (2016), 22 pages. 1

[13] M. H. Tahir, G. M. Cordeiro, M. Mansoor, M. Zubair, The Weibull-Lomax distribution: properties and applications, Hacet. J. Math. Stat., 44 (2015), 455-474. 4.1, 4.2

[14] H. Torabi, N. H. Montazari, The logistic-uniform distribution and its application, Comm. Statist. Simulation Comput., 43 (2014), 2551-2569. 1 